\title{
Puerperal and Intrapartum Group A Streptococcal Infection
}

\author{
Eyal Y. Anteby, ${ }^{1 *}$ Simcha Yagel, ${ }^{1}$ Josef Hanoch, ${ }^{1}$ Mervyn Shapiro, ${ }^{2}$ \\ and Allon E. Moses ${ }^{2}$ \\ ${ }^{1}$ Department of Obstetrics and Gynecology, Hadassah University Hospital, Mt. Scopus, \\ Jerusalem, Israel \\ ${ }^{2}$ Department of Clinical Microbiology and Infectious Diseases, Hadassah University Hospital, Mt. \\ Scopus, Jerusalem, Israel
}

\begin{abstract}
Objective: To determine the demographic and clinical variables characteristic of non-epidemic intrapartum or puerperal group A streptococcal (GAS) infection.

Methods: The records of 47 patients diagnosed with intrapartum or puerperal GAS infection over a 6 11/2 year period at Hadassah-University Hospital-Mt. Scopus, Jerusalem were reviewed. Data regarding 25,811 women, the general population of women that delivered during that period, were obtained from their computerized medical records. Frequency distributions, $t$-test, chi-square, and Spearman's Rank Correlation were used, as appropriate, to analyze and compare demographic and clinical variables associated with development of GAS infection, its clinical course and subsequent development of septic shock.

Results: Mean age of mothers with GAS infection was higher than that of our general pregnant population (30.4 versus 27.4 years, $P=0.0019$ ), and a higher proportion of GAS infected patients (30\% versus $12 \%, P<0.005$ ) experienced PROM. Thirty-one (66\%) women had fever as their sole presenting symptom, eight (17\%) had fever and abdominal pain, seven (15\%) had fever and abnormal vaginal bleeding, and one patient $(2 \%)$ presented with a rash. Three patients $(6 \%)$ developed a septic shock. Two of these patients presented with symptoms more than 14 days after delivery.

Conclusions: We describe the characteristics of non-epidemic intrapartum or puerperal GAS infection. Data from our study and review of the literature suggest that some patients who develop septic shock may present later in the puerperium than patients with an uncomplicated GAS infection. Infect. Dis. Obstet. Gynecol. 7:276-282; 1999. @ 1999 Wiley-Liss, Inc.
\end{abstract}

G roup A streptococcus (GAS) is a cause of major morbidity and mortality throughout the world. The resurgence of this organism has reversed the previously marked decrease in incidence of GAS caused intrapartum and puerperal infections, ${ }^{1,2}$ GAS bacteremia and streptococcal toxic shock syndrome $^{1}$ and acute rheumatic fever, a post infection sequela which is the leading cause of acquired heart disease in young people in developing countries. ${ }^{2,3}$ Reports of several cases of necrotizing fasciitis have also served to focus attention on determining the causes of the increased incidence of severe GAS diseases. Because GAS penicillin resistance has not been documented, increased virulence of the GAS organism has been suggested. ${ }^{4}$

Previous reports on GAS-caused intrapartum

*Correspondence to: Eyal Y. Anteby, MD, Department of Obstetrics and Gynecology, Hadassah University Hospital, Mt. Scopus, Jerusalem, Israel. E-mail: anteby@cc.huji.ac.il 
and puerperal infections include: (a) two studies of life-threatening puerperal infection; ${ }^{5,6}$ (b) a single case of puerperal group A streptococcal infection; ${ }^{7}$ (c) four cases of group A streptococcal bacteremia; 8 and, (d) epidemics of "childbed fever." 9 The objective of this study was to identify factors characteristic of non-epidemic puerperal group A streptococcal infection. We retrospectively analyzed the charts of 47 patients in the obstetrics and gynecology department of Hadassah University Hospital, Mt. Scopus, Jerusalem, who, over a six and a half year period, June 1987 through December 1994, were diagnosed with intrapartum or puerperal group A streptococcal infection. Data regarding the general population of women giving birth during that period was extracted from their computerized medical records.

\section{SUBJECTS AND METHODS Patient Selection}

During June 1987 through December 1994, 25,811 women gave birth at Hadassah-University Hospital, Mt. Scopus in Jerusalem. Review of their records revealed that 3,403 patients were suspected of having intrapartum or puerperal infection, and 47 patients $(1.4 \%)$ were diagnosed with GAS infection. We analyzed these patients charts in order to determine the demographic and clinical variables characteristic of non-epidemic intrapartum or puerperal group A streptococcal infection.

\section{Definitions}

- Suspected intrapartum or puerperal genital tract infection-oral temperature $>38^{\circ} \mathrm{C}$ with no other identifiable source or unusual abdominal pain or late postpartum hemorrhage, all $\leq 50$ days postdelivery.

- Diagnosis of intrapartum or puerperal GAS infection-cervical swab positive for GAS in patient suspected of having intrapartum or puerperal infection.

- Premature rupture of membranes (PROM)rupture of membranes before the onset of labor.

- Hours post-delivery at which signs and/or symptoms appeared-(a) in instances of patient having not yet been discharged from the hospital: the number of hours post-delivery when signs and/or symptoms were reported by patient and/or noted by hospital staff; (b) in instances of postpartum patient having been discharged from the hospi- tal: the number of hours post-delivery when patient presented to emergency room with signs and/or symptoms. The number of hours postdelivery presentation with signs and/or symptoms in instances of either (a) or (b) is not necessarily the same as number of hours at actual onset of signs and/or symptoms.

\section{Cultures and Group A Streptococcus Determination}

Cervical cultures were performed for the 3,403 patients suspected of having intrapartum or puerperal infection. Urine cultures were conducted for 36 of the 47 GAS infection patients, blood cultures for 35 and throat cultures for 5. Group A streptococcus was identified from cultures which grew Bhemolytic colonies sensitive to bacitracin and positive for group A carbohydrate antigen on a latex agglutination test. ${ }^{10}$

\section{Chart Review}

Charts of those patients (47) having been diagnosed with intrapartum or puerperal GAS infection were reviewed and data regarding the following variables recorded for analysis: age at delivery, gravidity, parity, type of delivery (cesarean section/ normal), time interval between delivery and patient presentation with symptom(s), PROM/not PROM, oral temperature on presentation, WBC on presentation, signs and symptoms in addition to fever on presentation, primary and subsequent treatments (if any), complications experienced during course of disease and their resolution.

Initial treatment consisted of oral antibiotics for $14(30 \%)$ of the patients: amoxicillin for 12 patients, cephalexin and cotrimoxazole each in one patient. For five of these patients whose clinical condition deteriorated, the regimen was changed to intravenous (IV) penicillin, ampicillin or a combination of ampicillin and gentamicin. Intravenous antibiotics were administered as initial treatment for $32(68 \%)$ of the patients: 19 with a combination of ampicillin, gentamicin and metronidazole; 5 with a combination of ampicillin, gentamicin and clindamycin; 5 with penicillin; 2 with cefazolin; and one with gentamicin and clindamycin.

Intravenous treatment was continued for two days after the fever had resolved, except in instances of positive blood cultures, in which case it was continued, generally for seven days. For one 
patient, culture results had not been received and no treatment administered; no data was recorded regarding late sequelae.

\section{Statistical Methods}

Frequency distributions were calculated for the following variables: per year proportion of patients developing GAS infection, age, gravidity, hours post-delivery at presentation with signs and/or symptoms, type of signs and/or symptoms on presentation, WBC count and fever on presentation. Comparison between patients who developed GAS infection and total patients delivering during the study period was conducted using the $t$-test for mean age and chi-square for incidence of primigravida, PROM, induction of labor and delivery by cesarean section. Patients with GAS infection who had undergone PROM and those who had not were compared using $t$-test for the variables of age, fever, WBC count, mean hours post-delivery of presentation, and chi-square for incidence of primigravida, and delivery by cesarean section. Spearman's Rank Correlation was used to determine association between continuous variables such as hours postdelivery at presentation with signs and/or symptoms and fever.

\section{RESULTS \\ Yearly Incidence of GAS Infection Over Six and One-Half Year Study Period}

This review of 25,822 deliveries over the six and one-half years (June 1987 to December 1994) found that the incidence of GAS infections per 1,000 births varied greatly from year to year: $0.33-3.16 /$ $1,000, P=0.0021$. We found no clustering of cases. A total of 3,403 (13.2\%) patients were suspected of having intrapartum or puerperal GAS infection. Forty-seven patients $(1.4 \%)$ of the suspected cases and $0.18 \%$ of the total patients delivering were subsequently diagnosed with GAS infection.

\section{Presentation With Signs and/or Symptoms of GAS Infection}

All (except one) of the patients who were later identified as having GAS infection either presented with fever or developed fever in the first 48 hours after admission. The fever was usually of sudden onset, and for $31(65 \%)$ this was the sole symptom. In addition to fever, 8 patients (17\%) had abdominal pain, generally, poorly localized; 7
(15\%) had heavy vaginal bleeding, and one had a diffuse erythematous rash.

The great majority, $39(83 \%)$ of patients who were identified as having GAS infection presented with symptoms within one week of delivery: $4(9 \%)$ before or during delivery; cumulatively $21(45 \%)$ within 48 hours of delivery; 34 (72\%) within 5 days and $39(83 \%)$ within 7 days post-delivery. The remaining $8(17 \%)$ presented with symptoms between 8 and 50 days post-delivery.

\section{Laboratory findings}

WBC: WBC counts for patients with GAS infection ranged from $5,300-27,000 / \mu \mathrm{L}$, with $66 \%$ between $5,000-15,000 / \mu \mathrm{L}, 29 \%$ between 15,300 and $19,400 /$ $\mu \mathrm{L}$, one patient $22,000 / \mu \mathrm{L}$ and another $27,000 / \mu \mathrm{L}$. Cultures: cervical cultures of 3,403 patients suspected of having intrapartum or puerperal infection resulted in $1,150(33.8 \%)$ positive for a bacterial infection and $47(1.4 \%)$ positive for GAS. In 3 of these 47 cultures both group A and group B streptococci were isolated. No other pathogenic bacteria were found in addition to group A streptococcus. Fourteen (39\%) of 36 urine cultures were positive for GAS, as were 5 (14\%) of 35 blood cultures, and one of the 5 throat cultures.

\section{Clinical Outcome}

There was clinical improvement within $1-4$ days following initiation of treatment in all patients except the three who developed septic shock, two of whom were discharged within 2 weeks and the third after 30 days.

Comparison between patients who developed GAS infection and all patients delivering is presented in Table 1. Age: The mean age of mothers who developed GAS infection was higher than that of all patients giving birth during the period studied. PROM: the proportion GAS infection patients who had experienced PROM was higher than that in the general population delivering. In $10.6 \%$ of the GAS infection patients, PROM occurred $\geq 24$ hours before delivery.

\section{Comparison of GAS Infection Patients With and Without PROM (Table 2)}

The patients who developed GAS infection and had experienced PROM presented at a mean of 32 hours post-delivery, a median of 24 hours, while those who had not experienced PROM presented 
TABLE I. Comparison of characteristics of patients who developed GAS infection with all patients delivering between 1987 and 1994

\begin{tabular}{|c|c|c|c|c|c|}
\hline \multirow[b]{2}{*}{ Characteristic } & \multicolumn{2}{|c|}{$\begin{array}{c}\text { Patients who } \\
\text { developed } \\
\text { GAS* } \\
\text { infection } \\
(\mathrm{N}=47) \\
\end{array}$} & \multicolumn{2}{|c|}{$\begin{array}{c}\text { All patients } \\
\text { delivering } \\
(\mathrm{N}=25,8 \mid \mathrm{I}) \\
\end{array}$} & \\
\hline & No. & $(\%)$ & No. & $(\%)$ & \\
\hline Age (mean yr) & 30.4 & & 27.4 & & $(P=0.0019)$ \\
\hline Primigravida & 10 & $(21.3)$ & 7485 & (29) & $\left(N^{\dagger}\right)$ \\
\hline PROM & 14 & $(29.8)$ & 3097 & (12) & $(P=0.005)$ \\
\hline Cesarean delivery & 6 & $(12.8)$ & 3123 & (12.1) & (NS') \\
\hline Induced labor & 4 & $(8.5)$ & 4362 & (16.9) & $\left(\mathrm{NS}^{\dagger}\right)$ \\
\hline \multicolumn{6}{|c|}{$\begin{array}{l}\text { *GAS - Group A streptococcus. } \\
\text { tNS = Not statistically significant. }\end{array}$} \\
\hline \multicolumn{6}{|c|}{$\begin{array}{l}\text { TABLE 2. Characteristics of PROM and non-PROM } \\
\text { patients who developed GAS infection }\end{array}$} \\
\hline Characteristic & $\begin{array}{l}\mathrm{PR} \\
(\mathrm{N}\end{array}$ & $\begin{array}{l}\text { OM } \\
=14) \\
\end{array}$ & $\begin{array}{l}\text { Non-P } \\
(\mathrm{N}=\end{array}$ & $\begin{array}{l}\text { ROM } \\
\text { 33) }\end{array}$ & $\begin{array}{l}\text { Statistical } \\
\text { significance }\end{array}$ \\
\hline Age (mean yr) & & .4 & 29. & & $\left(\mathrm{NS}^{*}\right)$ \\
\hline Primigravida & & $.1 \%)$ & $9(25$. & 7\%) & (NS*) \\
\hline \multicolumn{6}{|l|}{ Hrs. to present } \\
\hline Cesarean delivery & $4(2$ & $3.6 \%)$ & $2(5$. & $7 \%)$ & $(P=0.04)$ \\
\hline Fever (mean) & & 3.6 & 38. & & $\left(N S^{*}\right)$ \\
\hline Septic shock & & $.1 \%)$ & $2(5$. & $7 \%)$ & (NS*) \\
\hline
\end{tabular}

*NS = Not statistically significant.

at a mean of 181 hours, a median of 96 hours. Although the median hours post-delivery of presentation for the non-PROM patients was four times that of the PROM patients, the difference did not achieve statistical significance $(P=0.06)$, very probably because of the relatively small sample in the study. Neither age, proportion of primigravidas, degrees fever, nor WBC count differed significantly between GAS infection patients who had and had not experienced PROM.

\section{Description of Unusual Cases}

Four patients had unusual clinical courses: one had a rash and three had septic shock. Three of the four patients described below (75\%), unlike eight of the total number of patients who developed GAS infection (17\%), presented with symptoms more than a week post-delivery.

\section{Fever with rash}

Three weeks after a normal vaginal delivery, a 35year-old, gravida 3, para 2, developed a maculo- papular rash on the face which spread to the trunk and extremities. Several days later, as the rash began to fade and desquamate, a previously unnoticed vaginal discharge appeared and she was treated with metronidazole. Thirty-two days postdelivery, fever developed and after another ten days, six weeks post-delivery, the woman presented to the emergency room with a fever of $38.7^{\circ} \mathrm{G}$, diffuse rash, desquamation of the hands, facial edema, green vaginal discharge and mild adnexal tenderness. Her blood pressure was normal. After three days of clinical improvement, she was discharged with no further treatment. A uterine cervical culture was eventually positive for GAS.

\section{Septic shock}

Three of the patients presented with or developed septic shock while hospitalized with GAS infection. The first, a 23-year-old primigravida, had been induced after 37 weeks of gestation because of pregnancy-induced hypertension. Forty-eight hours after an otherwise normal delivery, she developed a fever of $38^{\circ} \mathrm{C}$ which resolved without treatment. Three weeks later, she presented to the emergency room with a fever of $39.7^{\circ} \mathrm{C}$ and malaise which had begun two days earlier. There was diffuse tenderness of the lower abdomen and uterus; pus exuded from the cervix; the WBC count was $16,200 / \mu \mathrm{L}$. Treatment with IV gentamicin and clindamycin was initiated. The day after admission, she became oliguric, her temperature increased to $40^{\circ} \mathrm{C}$, and she became hypotensive with a pulse rate of $120 / \mathrm{min}$. The cervical culture was positive for group A streptococcus. Over the next three days, the patient improved and was discharged without sequelae.

The second, a 24-year-old, gravida 3, para 3, had been delivered by cesarean section at 32 weeks gestation, subsequent to PROM. She presented to the emergency room 48 days after delivery with a fever of $39.5^{\circ} \mathrm{C}$, nausea, vomiting, diarrhea and lower abdominal pain. The abdominal examination was unremarkable, white blood count was 15,100 / $\mu \mathrm{L}$ and moderate ascites was observed by an ultrasonographic examination. Although uterine cervical cultures were taken, she was discharged because her clinical condition had improved over the next few hours and her temperature had dropped spontaneously. Twelve hours later she returned with diffuse abdominal pain, fever of $38^{\circ} \mathrm{C}$, blood 
pressure of $90 / 60 \mathrm{~mm} / \mathrm{Hg}$, pulse rate of $120 / \mathrm{min}$. The abdominal examination was consistent with peritonitis and exploratory laparotomy was performed. During this procedure $1,500 \mathrm{cc}$ of pus were evacuated from the abdominal cavity. The uterus and ovaries showed signs of inflammation but there was no evidence of necrosis. Thereafter, she was hypotensive and developed an adult respiratory distress syndrome (ARDS). Blood, cervical and abdominal cultures were positive for GAS. Initial treatment consisted of IV ampicillin, gentamicin and metronidazole was later changed to penicillin, dopamine and positive end expiratory pressure. The patient recovered gradually and was discharged after 14 days.

The third patient was a 29 -year-old, gravida 2 , para 1, who experienced diffuse abdominal pain the day after having been discharged following a normal vaginal delivery. She presented for admission the next day (four days post-delivery), with a temperature of $37.1^{\circ} \mathrm{C}$, pulse rate of $150 / \mathrm{min}$, blood pressure of $100 / 50 \mathrm{~mm} \mathrm{Hg}$, hemoglobin of $11.4 \mathrm{gr} \%$, WBC count of $15,800 / \mu \mathrm{L}$. and a diffusely distended and tender abdomen. A computerized tomography scan showed moderate ascitic and pleural effusion and colonic distention. The WBC count increased to $40,000 / \mu \mathrm{L}$ with platelets at $1,300 \times 10^{3} / \mathrm{ml}$. Uterine cervical and blood cultures were positive for GAS. Treatment consisted of IV ampicillin, gentamicin and metronidazol. The patient improved slowly, and was discharged after 28 days.

\section{DISCUSSION}

Our retrospective study of 25,822 deliveries at Hadassah Hospital, Mt. Scopus in Jerusalem over a six and a half year period showed that 3,403 were suspected of having intrapartum or puerperal infection. Forty-seven patients, $0.18 \%$ of the total patient population and $1.38 \%$ of patients suspected of having intrapartum or puerperal infection, had group A streptococcal (GAS) infection. Four percent of all our positive cervical cultures were GAS. Beargie et al. ${ }^{12}$ found that $0.28 \%$ of asymptomatic parturients admitted to the delivery room carried GAS. Other studies report the rate for GAS in patients with puerperal endometritis as $1-3 \% .^{7,13,14}$ Although typically, the patient with GAS infection presents with fever as the sole symptom with no localizing signs on physical examination as did $66 \%$ of our patients, there can be additional symptoms such as abdominal pain or heavy vaginal bleeding which $34 \%$ of our GAS infection patients experienced. In addition, the lochia of patients with streptococcal infection is usually sero-sanguinous and non-foul. ${ }^{7,9} \mathrm{We}$ found that in all cases except the three in which septic shock developed, clinical improvement was effected within $1-4$ days following institution of antibiotic treatment. Penicillin, erythromicin and clindamycin are the drugs of choice. ${ }^{15}$ Our findings suggest that initial intravenous antibiotic treatment of GAS puerperal infection may be superior to oral treatment, as a third of the patients initially administered oral antibiotic required a shift to intravenous therapy.

\section{Factors Characteristic of Non-Epidemic Intrapartum or Puerperal GAS Infection}

A number of factors were identified as characteristic of non-epidemic intrapartum or puerperal GAS infection. The mean age of the patients who developed GAS infection was statistically significantly higher than that of all women giving birth during the study period, even though there were a similar proportions of primi to multigravidas in both groups. Labor had been induced in patients who developed GAS infection at slightly greater than half the rate $(8.5 \%)$ as total patients delivering (16.9\%). It can be conjectured that incipient infection triggered a somewhat earlier delivery in the patients who were subsequently diagnosed with GAS infection than in the overall population delivering. Indeed, a statistically significantly higher proportion of patients who developed GAS infection had experienced PROM (29.8\%) than had the total patients delivering (12\%) and those who had experienced PROM presented with symptoms of GAS infection at a median of one-fourth the hours post-delivery as non-PROM GAS infection patients. In $10.6 \%$ of the GAS infection patients, PROM occurred $\geq 24$ hours before delivery, a rate that correlates with $13.3 \%$ found in a study of 48 streptococcal bacteremias. ${ }^{5}$ All (except one) of the patients who developed GAS infection presented with fever. Thus, we emphasize the need for thorough evaluation and follow-up of all patients with post-delivery fever, including performing cervical and blood cultures so as to determine whether intrapartum or puerperal group A streptococcus is present. 


\section{Unusual Clinical Course}

The clinical course in four of our patients took an unusual direction: one woman had a diffuse erythematous rash and three developed septic shock. In three of the four, onset of symptoms, unlike the great majority of patients, was more than a week after delivery. The patient who presented with a desquamating rash may have had an exotoxin Aproducing strain of GAS which caused her mild scarlet fever like illness which did not meet the criteria for streptococcal toxic shock syndrome and which resolved spontaneously.

We reviewed the characteristics of the three patients in our study and four from two other investigations, ${ }^{1,2}$ who developed septic shock. The interval between delivery and onset of shock varied: two patients presented within 48 hours postdelivery, one, 4 days following delivery, and three between 2-6 weeks postpartum. Initial symptoms were high grade fever in six of the seven women; the patient who presented with normal temperature experienced diffuse abdominal pain. Manifestations of septic shock-hypotension, tachycardia, tachypnoea-were evident immediately upon arrival in three patients: in two of these three patients there was acute renal failure (ARF), hemolysis in two patients and, in one patient each acidosis and rash. One of these patients had been suffering from flu-like symptoms for one week prior to admission. Septic shock symptoms developed one day after admission: ARDS ( 2 patients), ARF, ${ }^{1}$ abdominal distention, ascites, pleural effusion, tachycardia and marked leukocytosis. ${ }^{1}$ In five of the patients there was purulent or serosanguinous ascites. All patients with septic shock received broad spectrum antibiotics. Three patients underwent a total abdominal hysterectomy, two with bilateral salpingo- and one with left salpingooophorectomy and one a subtotal hysterectomy with bilateral salpingo-oophorectomy. Neither a hysterectomy nor oophorectomy was performed on any of our patients. Both adnexal necrosis and pelvic vessel thrombosis were seen in two patients, probably representing a more fulminant course of disease. One of these patients was the only patient to have died.

Although few cases of severe GAS puerperal infection have been reported and the infection may initially seem poorly manifested, it can, neverthe- less, become overwhelming, and in rare cases, lead to death. This type of presentation is typical of GAS infection and presents similarly at other sites, for example, the skin. Therefore, it behooves the obstetrician to be alert to the possibility of a rapidly developing infection.

Late onset of GAS infection (between 8-50 days post-delivery) occurred in eight women in our study, four of whom either presented atypically or experienced a clinically complicated course suggesting that late onset puerperal GAS infection may represent a different entity from earlier onset infection. Prolonged subclinical infection may be a causal factor in an unusual clinical course. Indeed, one day post-delivery, two of these patients had had low grade fever which had resolved without treatment.

\section{ACKNOWLEDGMENT}

We thank Dr. Nina Geller for her critical review of the manuscript.

\section{REFERENCES}

1. Stevens DL, Tanner MH, Winship J, Swarts R, Ries KM, Schlievert PM, et al. Severe group A streptococcal infections associated with a toxic shock-like syndrome and scarlet fever toxin A. N Eng J Med 1989;321:1-7.

2. Veasy GL, Wiedmeier SE, Orsmond GS, Ruttenberg HD, Boucek MM, Roth SJ, et al. Resurgence of acute rheumatic fever in the intermountain area of the United States. N Engl J Med 1987;316: 421-427.

3. Wald ER, Dashefsky B, Feidt C, Chiponis D, Byers C. Acute rheumatic fever in western Pennsylvania and the tristate area. Pediatrics 1987;80:371-374.

4. Moses AE, Ziv A, Harari M et al. Increased incidence and severity of Streptococcus pyogenes bacteremia in young children. Ped Inf Dis J 1995;14(9):767-770.

5. Silver RM, Heddleston LN, McGregor JA, Gibbs RS. Life-threatening puerperal infection due to group A streptococci. Obstet Gynecol 1992;79:894-896.

6. Nathan L, Peters MT, Ahmed AM, Leveno KJ. The return of life-threatening puerperal sepsis caused by group A streptococci. Am J Obstet Gynecol 1993;169: 571-572.

7. Ledger WJ, Headington JT. Group A B-hemolytic streptococcus, an important cause of serious infections in obstetrics and gynecology. Obstet Gynecol 1972;39(3): $474-482$.

8. Gibbs RS, Blanco JD. Streptococcal infections in pregnancy, a study of 48 bacteremias. Am J Obstet Gynecol 1981;140:405-411.

9. McGregor J, Ott A, Villard M. An epidemic of "Childbed fever." Am J Obstet Gynecol 1984;150:385-388.

10. Bisno AL, Streptococcus pyogenes. In: Mandell GL, Doug- 
las RG Jr., Bennett JE, editors. Principles and Practice of Infectious Diseases. 4th ed. New York, 1995, p 17861799.

11. Gunn GC, Mishell DR Jr, Morton DG. Premature rupture of the membranes: a review. Am J Obstet Gynecol 1970;106:469.

12. Beargie R, Lynd P, Tucker E. Perinatal infection and vaginal flora. Am J Obstet Gynecol 1975; 122:31-33.
13. Gibbs RS, Jones PM, Wilder CJ. Antibiotic therapy of endometritis following cesarean section. Obstet Gynecol 1978; 52:31.

14. Gilstrap LC, Cunningham FC. The bacterial pathogenesis of infection following cesarean section. Obstet Gynecol 1979;53:545.

15. Stevens DS. Invasive group A streptococcal infection. Clin Infect Dis 1992;14:2-13. 


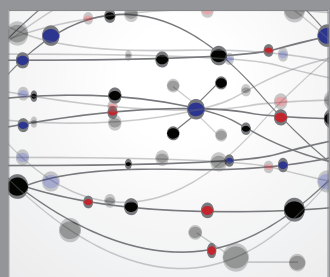

The Scientific World Journal
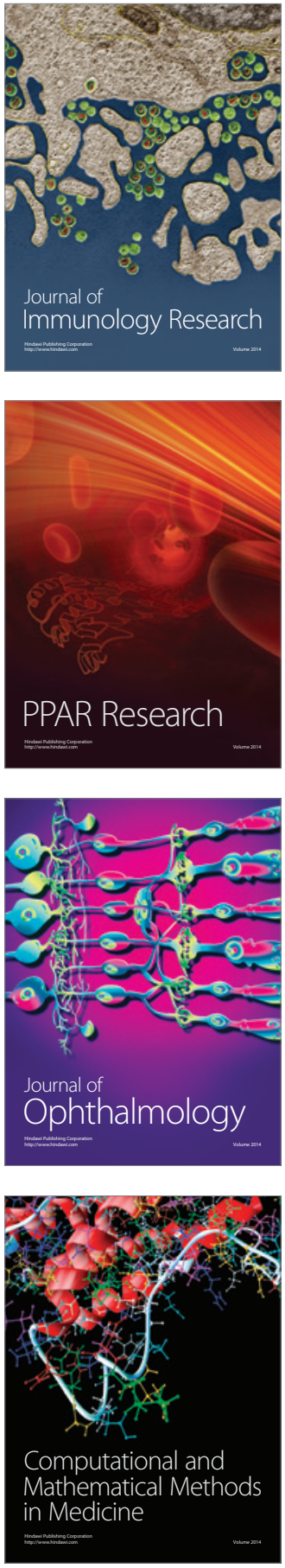

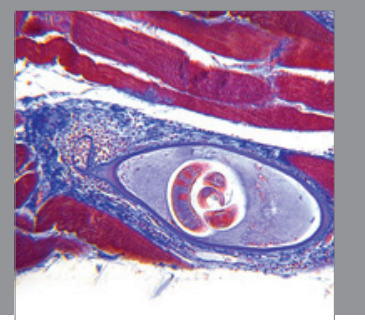

Gastroenterology

Research and Practice
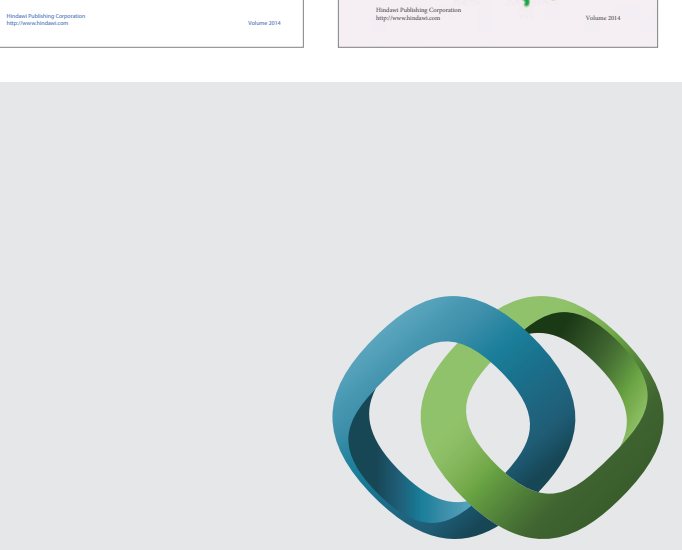

\section{Hindawi}

Submit your manuscripts at

http://www.hindawi.com
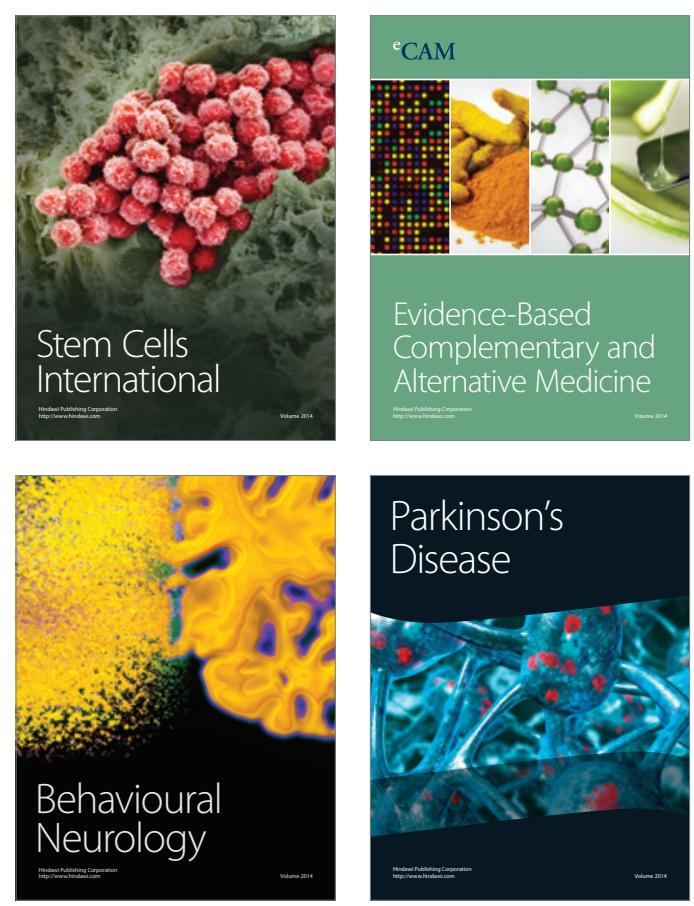

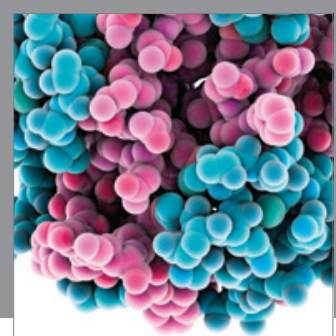

Journal of
Diabetes Research

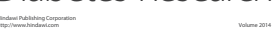

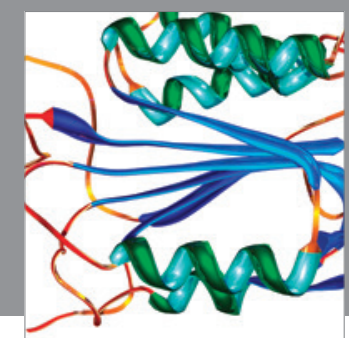

Disease Markers
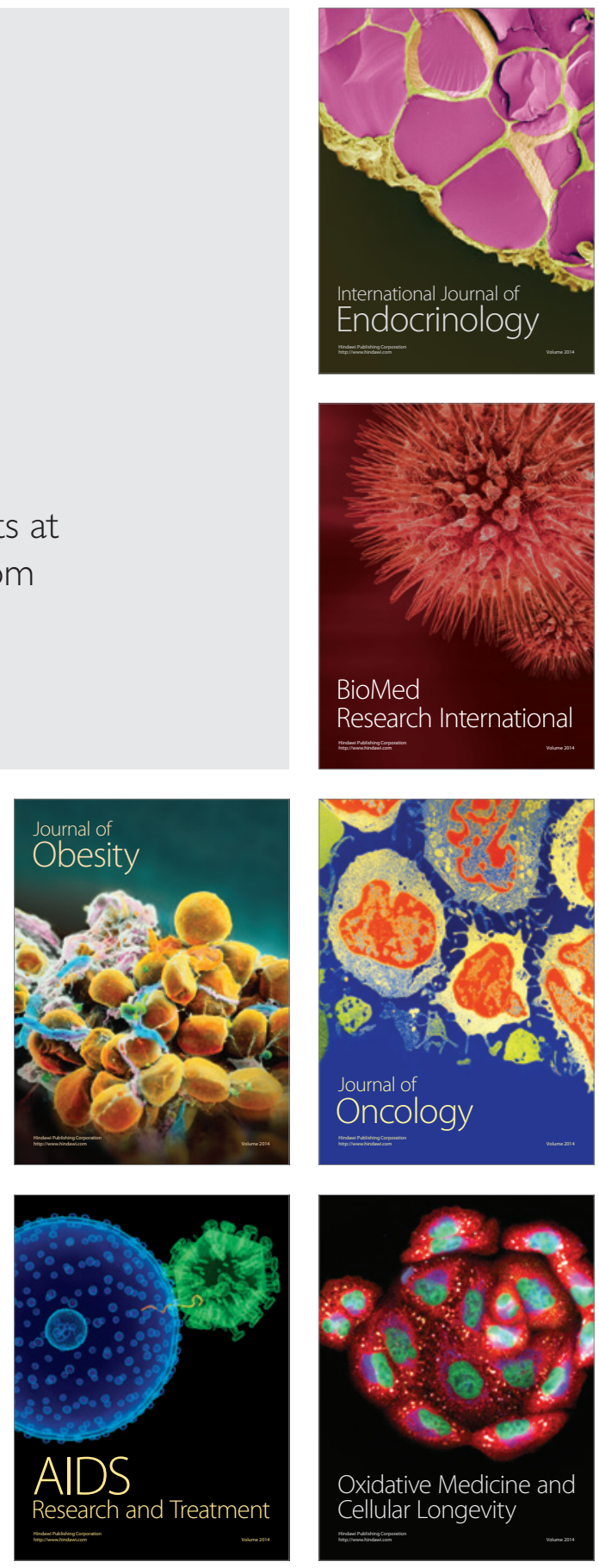